\title{
An Extremely Rare Case of Huge Oncocytic Adenolipoma of Parotid Gland
}

\author{
Dipesh Shakya ${ }^{1}$ and Ajit Nepal ${ }^{2}$ \\ ${ }^{1}$ Civil Service Hospital \\ ${ }^{2}$ Patan Academy of Health Sciences School of Medicine
}

May 26, 2020

\begin{abstract}
Oncocytic adenolipoma is a rare tumor composed of adipose tissue and oncocytic epithelial cells in different proportions presenting in the salivary gland. Until now, less than 20 such cases are reported. We report another such rare case presented as huge neck mass.
\end{abstract}

\section{Key Clinical Message}

Oncocytic adenolipoma is a rare tumor composed of oncocytic epithelial cells and adipocytes presenting in the salivary gland. Until now, less than 20 such cases are reported. We report this a rare case presented as a huge mass from the parotid gland.

\section{Introduction}

Lipomatous tumors are one of the commonly seen tumors of subcutaneous soft tissue. However, those originating from the salivary gland are extremely rare. Oncocytic adenolipoma or lipoadenoma is one of the rare biphasic lipomatous tumors which is composed of oncocytes admixed with mature adipocytes. ${ }^{1}$ The first case of oncocytic adenolipoma of the submandibular gland was reported by Hirokawa et al. in $1998 .^{2}$ Only the case reports are published in the literature due to its rarity. In the 2005 World Health Organization (WHO) histologic classification of tumors of salivary glands, this tumor was not included. ${ }^{3}$ We report a case of a patient with a huge painless mass in the neck with a history of more than 18 years of swelling and underwent excision. The case was followed up for 5 years post-surgery to rule out excision. The paper is presented to consider for this rare differential diagnosis for neck mass.

\section{Case Presentation}

A 46 years old man from remote and difficult to reach part of the country, came to our center with complaints of huge neck mass in the right side, almost occupying the whole of the right neck from level 2,3,4 and 5 . He gave a history of swelling, which was gradually progressive and painless for more than 18 years. Due to financial reasons and remote parts of the country, the patient presented very late after the onset of disease. Late presentation to the hospital is a common problem in developing countries. But recently, for the past 2 years, the size of the swelling has increased progressively than before. With the fear of cancer, the patient somehow managed to turn up in the hospital. There was no history of xerostomia, dysphagia, shortness of breath, facial deviation, change in sensation, fever, no intraoral discharge, loss of weight or change in appetite, and no known comorbidity.

On examination, the mass was well defined, multilobulated with a size of approximately $15 \times 11 \mathrm{~cm}$ at the right side of neck, superiorly up to the level of ear lobule, inferiorly $4 \mathrm{~cm}$ above the clavicle, medially up to midline and posterolaterally occupying half of posterior triangle (Figure 1). The overlying skin was free, 
with no pain or tenderness on palpation, and the mass was firm in consistency, mobile, and, multilobulated with a well-defined border. There was no change in skin color, no sinuses, and no scar. Based on history and examination, a provisional diagnosis of soft tissue mass probably salivary gland origin was made. Fine needle aspiration cytology was done, which could not provide a definite opinion and just gave a suggestion of fat origin.

CT scan was done, which reported as a huge lobulated mass measuring $15 \times 10 \times 6.5 \mathrm{~cm}$ in the right side of the neck and face. Radiologically the mass contained enhancing solid areas on the periphery, which was supplied by large vessels and had fat components medially. No calcification or cystic areas noted and no significant lymph nodes. The lesion was abutting the parotid and submandibular gland. The CT reported lesion to be suggestive of the fat-containing soft-tissue tumor as angiolipoma with a differential of liposarcoma. (Figure 2)

The vertical incision was given on the right side over the swelling. The subplatysmal flap was elevated. The capsule of the mass was dissection from all around the margin superiorly and inferiorly. The dissection was carried on securing the hemostasis. There were no findings suggestive of malignancy such as adhesions, friability of tissues, or invasion of surrounding tissues. Medially the tissue was abutting the lower pole of the superficial lobe of the parotid gland. The mass of excised in toto and sent for histopathological examination. The drain was kept, and the surgical site was sutured. (Figure 3 and 4)

Gross examination revealed a single piece of tissue comprising of two nodular tissue attached in the center by fibrofatty tissue measuring together $15 \times 9.8 \times 5 \mathrm{~cm}$. The outer surface was nodular, brownish with congested vessels which were capsulated with pericapsular fat. Cut surface showed homogeneous brownish (mahogany brown) lobulated areas admixed with fatty tissue.

Microscopic examination showed multiple lobules of tumor separated by thin fibrovascular septa with a fibrous capsule. The lobules composed of prominent oncocytes arranged in tubules, admixed with fatty tissue composed of mature adipocytes in varying proportions. Foci of squamous and sebaceous differentiation, chronic inflammatory cells, and stromal edema were evident as well. No features of malignancy noted. The final diagnosis of oncocytic adenolipoma of parotid gland origin was made.

\section{Discussion}

Lipomatous tumors comprise $0.5 \%$ of salivary gland tumors, with the most common site being the parotid, followed by the submandibular gland. ${ }^{1}$ Histologically its spectrum can range from pure lipomatous neoplasm like the one seen in the cutaneous site or can be admixed with epithelial component specific to salivary gland. ${ }^{3}$

Thus, it can be of monophasic (only lipomatous component) or biphasic (epithelial plus lipomatous component) histological type. Among the biphasic type, the epithelial component can be of oncocytic or nononcocytic. ${ }^{1}$ Oncocytic adenolipoma is an extremely rare benign tumor of salivary glands which consists of oncocytic epithelial components admixed with mature adipocytes in varying proportion. ${ }^{4}$ When Chi et al. reported in 2015, only 18 such cases were reported in the literature. ${ }^{5}$ This rare condition has been published mainly as single case presentation in the literature under different names as oncocytic sialipoma, oncocytic lipoadenoma, and adenolipoma. ${ }^{3}$

Most of the cases in the literature revealed that the oncocytic adenolipoma presented as a slowly growing, asymptomatic swelling most commonly from the parotid gland. The other areas where adenolipoma can be found include breast, thyroid, parathyroid, and skin. ${ }^{6}$ The duration of symptoms ranged from 15 days to 11 years. ${ }^{7}$ However, in our case, the history of onset was more than 18 years. Such a long duration has not been reported before. The age of onset for the condition has been widely reported from 7 to 89 years old, but mostly seen in adults with a mean age of 57.7 years old. ${ }^{8}$ There are literature stating equal sex predilection to being slight male predominance of 2-4:1.3 The treatment usually consists of excision with no risk of recurrence or aggressive behavior.$^{8}$

The pathogenesis of the lesion is still elusive. There are different speculations regarding it. One theory given 
by Nagao stated it as an entrapment of glandular component during lipomatous proliferation. He suggested it being not of neoplastic origin but as a type of lipoma rather than hamartomatous origin. ${ }^{9}$ However, Parente et al. found the disorganized proliferation of neural and vascular structures by which they gave a theory of hamartomatous origin. ${ }^{10}$ The hypothesis of the hamartomatous nature given by Parente et al. was negated by Akrish et al., who proposed another theory. Their theory suggested that it was the dysfunction of the salivary gland, leading to a modification of the normal gland function that leads to the tumor. To support this theory were the reasons as prolonged history, lack of recurrence after excision, and histological features as ductal ectasia, periductal fibrosis, oncocytic metaplasia and replacement of glandular tissue with mature adipocytes and chronic inflammatory cells. ${ }^{11}$

Clinically, the differential diagnosis of the present case with a huge lesion might include varieties of the neck mass, including all benign lesions of the salivary gland. Radiological investigation of CT scan helped us to narrow down the differential to the fat-containing lesion as lipoma to lipoadenoma. Except for the huge size of the mass, the radiologically, the lesion was not that suggestive of being malignant type. However, FNA cytology was not found to be helpful for us. Histologically, the differential diagnosis of oncocytic adenolipoma includes all other lesions containing oncocytes, such as oncocytoma, oncocytic carcinoma, Warthin's tumor, and oncocytic cystadenoma, as well as oncocytic variants of pleomorphic adenoma, myoepthelioma, mucoepidermoid carcinoma, and epithelial-myoepithelial carcinoma. ${ }^{6,8}$ The excisional surgery was done as reported in almost all cases reported in the literature. ${ }^{1,2,5,7}$ We followed the patient for five years, and until now, no recurrence of the lesion was noted. The present case is reported with the aim to make aware of the rare possibility of this extremely rare lipomatous lesion of the salivary gland in a patient presenting with a huge neck mass. We also aimed to confirm the long-term disease-free interval of 5 years after the surgical resection in this lesion.

\section{Conclusion}

Oncocytic adenolipoma is a unique, extremely rare salivary gland neoplasm that one needs to be aware of and should also be regarded as one of the differential diagnoses in the patient presenting with any huge neck mass. Given the rarity, we describe this case to generate awareness and add to the few reported cases in the literature.

\section{Author Contribution}

DS: Conceptualization and preparation of manuscripts

AN: Critically reviewed the manuscript

\section{References}

1. Parmar HV. Oncocytic lipoadenoma of submandibular gland: a case report. J Clin Diagn Res . 2015;9(3): ED05-ED6. doi:10.7860/JCDR/2015/11291.5693

2. Swathi V, Chandrasekhar HR. Oncocytic lipoadenoma of parotid gland with sebaceous differentiation. Ind J Pathol Oncol, 2018;5(3):516-517. doi: 10.18231/2394-6792.2018.0100

3. Agaimy A. Fat-containing salivary gland tumors: a review. Head Neck Pathol . 2013;7 Suppl 1(Suppl 1): S90-S96. doi:10.1007/s12105-013-0459-7

4. Seth E. Pross, Jolie L. Chang, Christine M. Glastonbury, Annemieke van Zante, Khaing Soe Win \& David W. Eisele. Characteristics of oncocytic lipoadenoma of the submandibular gland: a case report, Acta Oto-Laryngologica Case Reports, 2016;1:1,4-7,doi:10.1080/23772484.2016.1167605

5. Chi CL, Kuo TT, Lee LY. Oncocytic lipoadenoma: a rare case of parotid gland tumor and review of the literature. J Pathol Transl Med . 2015;49(2):144-147. doi:10.4132/jptm.2014.02.10

6. Devadoss, C. W., Murugan, P., Basu, D., \& Jagdish, S. Oncocytic lipoadenoma of the parotid gland: Report of a rare case. Journal of Clinical and Diagnostic Research .2012; 6 (6): 10761078. https://doi.org/JCDR/2012/4526:2332

7. Mitsimponas KT, Agaimy A, Schlittenbauer T, Nkenke E, Neukam FW. Oncocytic lipoadenoma of the parotid gland: a report of a new case and review of the literature. Int J Clin Exp Pathol . 
2012;5(9):1000-1006.

8. Lau SK, Thompson LD. Oncocytic lipoadenoma of the salivary gland: a clinicopathologic analysis of 7 cases and review of the literature. Head Neck Pathol . 2015;9(1):39-46. doi:10.1007/s12105-014-0543-7

9. Nagao T, Sugano I, Ishida Y, et al. Sialolipoma: a report of seven cases of a new variant of salivary gland lipoma. Histopathology . 2001;38(1):30-36. doi:10.1046/j.1365-2559.2001.01054.x

10. Parente P, Longobardi G, Bigotti G. Hamartomatous sialolipoma of the submandibular gland: case report. Br J Oral Maxillofac Surg . 2008;46(7):599-600. doi:10.1016/j.bjoms.2008.02.006

11. Akrish S, Leiser Y, Shamira D, Peled M. Sialolipoma of the salivary gland: two new cases, literature review, and histogenetic hypothesis. J Oral Maxillofac Surg . 2011;69(5):1380-1384. doi:10.1016/j.joms.2010.05.010
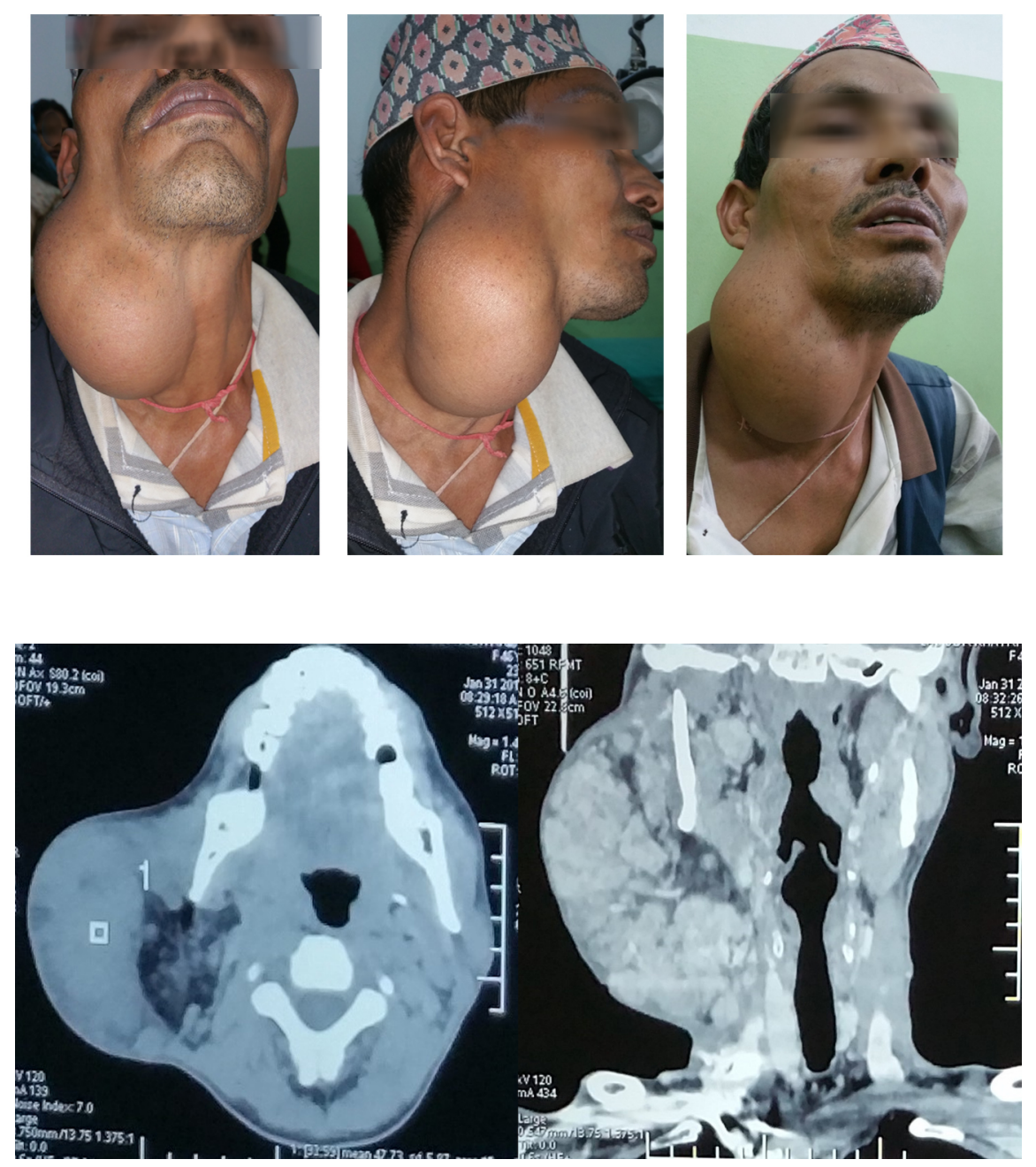

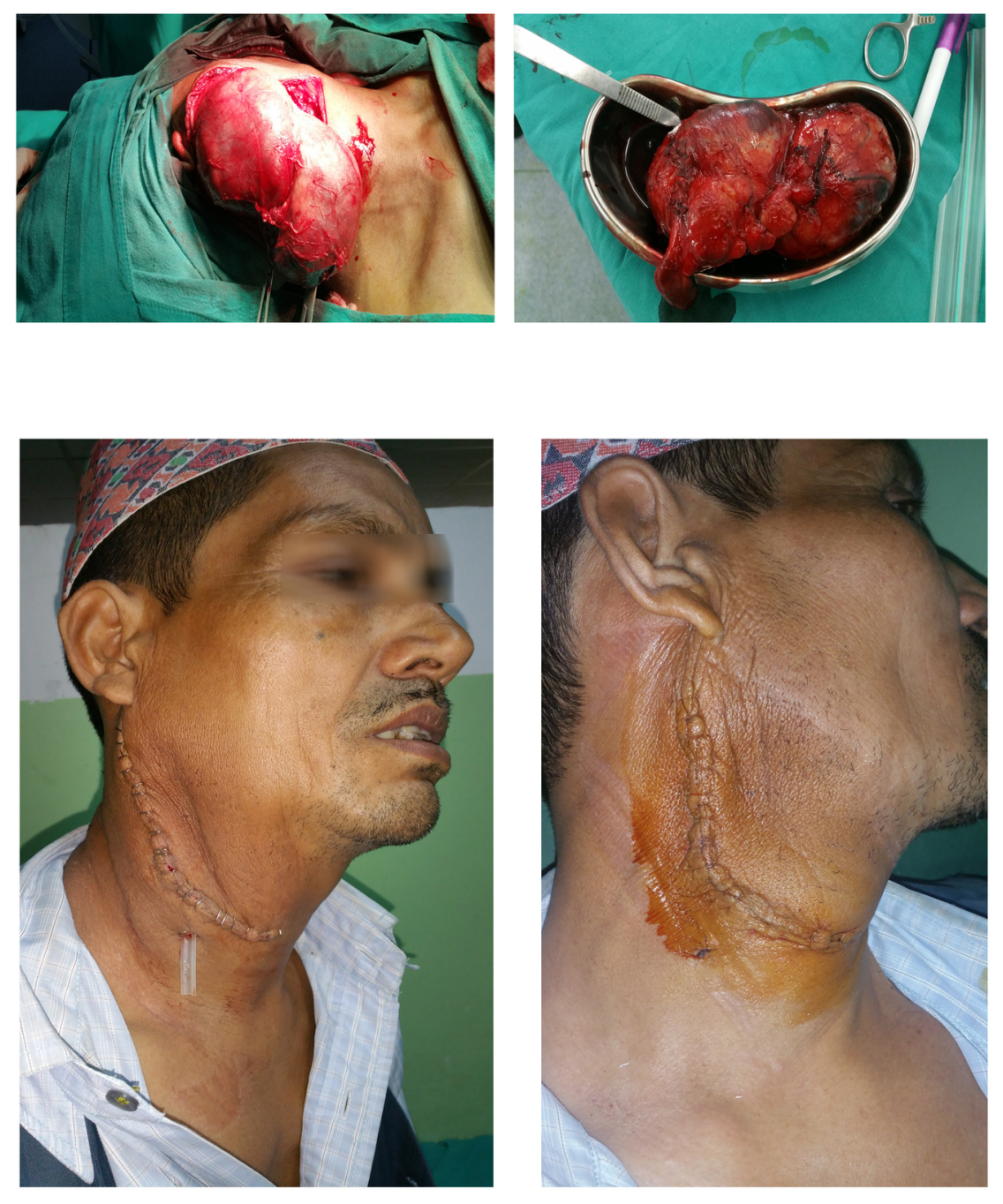